\title{
EXTENSION THEORY FOR CONNECTED HOPF ALGEBRAS
}

\author{
BY WILLIAM M. SINGER
}

Communicated by Michael Artin, April 3, 1970

1. Introduction. Let $K$ be a fixed commutative ring with unit. We will deal with graded algebras, coalgebras, and Hopf algebras over $K$ as defined in Milnor-Moore [4], but we assume that the underlying $K$-modules are connected.

Suppose $A, B$ are Hopf algebras; $A$ commutative and $B$ cocommutative. By an extension of $B$ by $A$ we mean a diagram of Hopf algebras and Hopf maps

$$
A \stackrel{\alpha}{\rightarrow} C \stackrel{\beta}{\rightarrow} B
$$

in which $C$ is isomorphic to $A \otimes B$ simultaneously as a left $A$-module and right $B$-comodule. In this paper we announce results which describe and classify all extensions by $B$ by $A$. Proofs will appear in [5].

2. Matched pairs. If $B$ is an algebra we write

$$
\eta: K \rightarrow B, \quad \mu_{B}: B \otimes B \rightarrow B
$$

for the unit and multiplication, respectively. If $A$ is a coalgebra we write

$$
\epsilon: A \rightarrow K, \quad \psi_{A}: A \rightarrow A \otimes A
$$

for the counit and comultiplication.

As the first step in classifying extensions, we will show in [5] how a diagram (1.1) determines a pair of $K$-linear maps

$$
\sigma_{A}: B \otimes A \rightarrow A, \quad \rho_{B}: B \rightarrow A .
$$

$\sigma_{A}$ is the "action" of base on fiber that one expects in an extension problem; $\rho_{B}$ is its dual. We prove:

(a) $\sigma_{A}$ gives $A$ the structure of a left $B$-module algebra;

(b) $\rho_{B}$ gives $B$ the structure of a right $A$-comodule coalgebra;

(c) the diagram commutes:

AMS 1969 subject classifications. Primary 1680, 1820; Secondary 5534.

Key words and phrases. Algebra, coalgebra, Hopf algebra, extensions of Hopf algebras, triples, cotriples. 


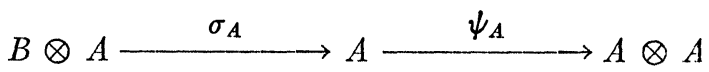

$$
\downarrow \psi_{B} \otimes \psi_{A}
$$

$B \otimes B \otimes A \otimes A$

$$
\mid \rho_{B} \otimes B \otimes \mathrm{A} \otimes \mathrm{A}
$$

$B \otimes A \otimes B \otimes A \otimes A$

$$
\lfloor(1,4,2,3,5)
$$

$B \otimes A \otimes A \otimes B \otimes A \stackrel{\sigma_{A} \otimes \mathrm{A} \otimes \sigma_{A}}{\longrightarrow} A \otimes A \otimes A$

(d) the diagram commutes:

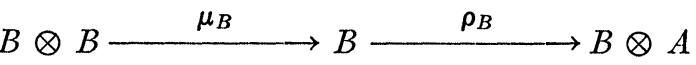

$$
\downarrow \psi_{B} \otimes B
$$

$B \otimes B \otimes B$

$$
\rho_{B} \otimes \mathrm{B} \otimes \rho_{B}
$$

$B \otimes A \otimes B \otimes B \otimes A$

$$
\downarrow(1,4,2,3,5)
$$

$B \otimes B \otimes A \otimes B \otimes A \stackrel{B \otimes B \otimes A \otimes \sigma_{A}}{\longrightarrow} B \otimes B \otimes A \otimes A$

Conversely, suppose given a pair of Hopf algebras $(A, B)$ with $A$ commutative and $B$ cocommutative, and suppose given $K$-linear maps $\sigma_{A}, \rho_{B}$ satisfying conditions $(\mathrm{a})-(\mathrm{d})$. Then we call $(A, B)$ a "matched pair".

3. Bimodules over a matched pair. Suppose $(A, B)$ is a matched pair. Suppose $N$ is simultaneously a left $B$-module under $\sigma_{N}: B \otimes N$ $\rightarrow N$, and a right $A$-comodule under

$$
\rho_{N}: N \rightarrow N \otimes A
$$

Then we call $N$ an $(A, B)$-bimodule if the diagram commutes: 

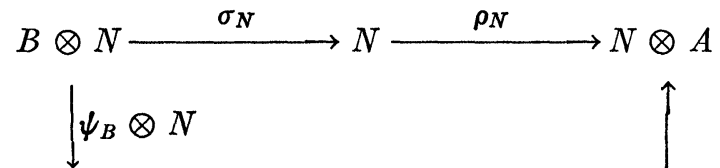

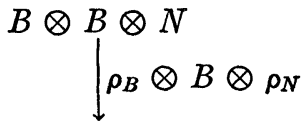

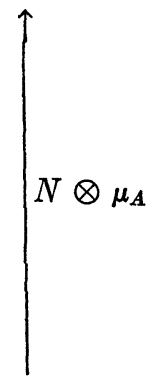

$B \otimes A \otimes B \otimes N \otimes A$
$\downarrow(1,4,2,3,5)$

$B \otimes N \otimes A \otimes B \otimes A \stackrel{\sigma_{N} \otimes A \otimes \sigma_{A}}{\longrightarrow} N \otimes A \otimes A$

For example, if $(A, B)$ is a matched pair we can give $A$ the structure of an $(A, B)$-bimodule, with left $B$-action $\sigma_{A}: B \otimes A \rightarrow A$, and right $A$-coaction $\psi_{A}: A \rightarrow A \otimes A$. A dual construction makes $B$ into an $(A, B)$-bimodule. We say $f: M \rightarrow N$ is a map of $(A, B)$-bimodules if $f$ is simultaneously a map of left $B$-modules and right $A$-comodules.

The interpretation of diagrams (2.1), (2.2), (3.1) is found in:

THEOREM 3.1. Let $N$ be a bimodule over the matched pair $(A, B)$. Let the $\operatorname{map} \tilde{\sigma}_{N} \otimes A: B \otimes N \otimes A \rightarrow N \otimes A$ be the composition $\left(N \otimes \mu_{A}\right)\left(\sigma_{N} \otimes A \otimes \sigma_{A}\right)(1,4,2,3,5)\left(\rho_{B} \otimes B \otimes N \otimes A\right)\left(\psi_{B} \otimes N \otimes A\right)$. Let the $\operatorname{map} \tilde{\rho}_{N} \otimes_{A}: N \otimes A \rightarrow N \otimes A \otimes A$ be $N \otimes \psi_{A}$. Then:

(a) $\tilde{\sigma}_{N \otimes A}$ gives $N \otimes A$ the structure of a left $B$-module;

(b) $\tilde{\rho}_{N \otimes A}$ gives $N \otimes A$ the structure of a right $A$-comodule;

(c) with these structure maps $N \otimes A$ is in fact an $(A, B)$ bimodule which we denote $N \tilde{\otimes} A$;

(d) $\rho_{N}: N \rightarrow N \tilde{\otimes} A$ is a map of $(A, B)$-bimodules.

Theorem 3.1 has a dual. Given an $(A, B)$-bimodule $M$, Theorem (3.1)* tells how to give $B \otimes M$ the structure of an $(A, B)$-bimodule, denoted $B \otimes M$, in such a way that $\sigma_{M}: B \underset{\sim}{\otimes} M \rightarrow M$ is a map of $(A, B)$ bimodules.

4. $(A, B)$-algebras and $(A, B)$-coalgebras. By an $(A, B)$-algebra we mean an $(A, B)$-bimodule $N$ that is also a commutative algebra, in such a way that $\mu_{N}: N \otimes N \rightarrow N$ is both a map of left $B$-modules and right $A$-comodules. $(A, B)$-coalgebras are defined similarly. For example if $(A, B)$ is a matched pair, then $A$ itself is an $(A, B)$-algebra, and $B$ is an $(A, B)$-coalgebra.

THEOREM 4.1. Let $N$ be an $(A, B)$-algebra. Let $N \tilde{\otimes} A$ have the algebra structure of the tensor product $N \otimes A$. Then $N \tilde{\otimes} A$ is an $(A, B)$-algebra, and $\rho_{N}: N \rightarrow N \tilde{\otimes} A$ is a map of $(A, B)$-algebras. 
Theorem 4.1 can be interpreted in the language of "triples" [1], [2]. Let $\Gamma$ be the category of $(A, B)$-algebras. Let $S: \Gamma \rightarrow \Gamma$ be the functor which carries $N$ to $N \tilde{\otimes} A$, and let $I: \Gamma \rightarrow \Gamma$ be the identity functor. Define functor transforms $\delta: I \rightarrow S, \sigma: S^{2} \rightarrow S$ by

$$
\delta(N)=\rho_{N}: N \rightarrow N \tilde{\otimes} A ; \quad \sigma(N)=N \otimes \epsilon \otimes A: N \tilde{\otimes} A \tilde{\otimes} A \rightarrow N \tilde{\otimes} A .
$$

Then $V \equiv(S, \delta, \sigma)$ is a triple on the category $\Gamma$.

Similarly, the dual of Theorem 4.1 gives a cotriple $W \equiv(T, d, s)$ on the category $\Delta$ of $(A, B)$-coalgebras. Here $T(M)=B \otimes M, d(M)$ $=\sigma_{M}: B \otimes \sim \rightarrow M$, and $S(M)=B \otimes \eta \otimes M: B \otimes M \rightarrow B \otimes B \otimes M$.

5. The cohomology of matched pairs. If $\phi$ is any category, write $S^{*}(\phi)$ for the category of cosimplicial objects over $\phi$, and $S_{*}(\phi)$ for the category of simplicial objects over $\phi$. Then the triple $V$ of $\$ 4$ gives rise in the usual way [1], [2] to a functor $V: \Gamma \rightarrow S^{*} \Gamma$; the cotriple $W$ gives rise to a functor $W: \Delta \rightarrow S_{*} \Delta$. For example, if $(A, B)$ is a matched pair, then $V(K)$ is the acyclic cobar construction on $A$, but it has some structure not present in the classical case ... an action of $B$ compatible with the coface operators. Similarly, $W(K)$ is the acyclic bar construction on $B$, with an $A$-coaction added.

If $M$ is an $(A, B)$-coalgebra and $N$ an $(A, B)$-algebra, let $\operatorname{Hom}_{(A, B)}(M, N)$ denote the set of maps of $(A, B)$-modules $f: M \rightarrow N$ for which $f_{0}: M_{0} \rightarrow N_{0}$ is the identity on $K$. Then $\operatorname{Hom}_{(A, B)}(M, N)$ is an abelian group under the composition law $f+g=\mu_{N}(f \otimes g) \psi_{M}$.

Now to any matched pair $(A, B)$ we associate a double cosimplicial abelian group $X(B, A)$ by setting:

$$
X^{p, q}(B, A)=\underset{(A, B)}{\operatorname{Hom}}\left(W(K)_{p}, V(K)^{q}\right) .
$$

Let $\bar{X}(B, A)$ be the associated "total" cochain complex. Then we define the cohomology of the matched pair $(A, B)$ by:

$$
H^{*}(B, A)=H^{*}(\bar{X}(B, A)) \text {. }
$$

6. Classification of extensions. Let $(A, B)$ be a matched pair under $\left(\sigma_{A}, \rho_{B}\right)$. Denote by $\operatorname{Opext}(B, A)$ the set of equivalence classes of extensions (1.1) which give rise to the given "matching." Our main result is:

THEOREM 6.1. There is a natural isomorphism:

$$
H^{3}(B, A)=\operatorname{Opext}(B, A) \text {. }
$$

7. Acknowledgments. The author's efforts have been sustained by 
James Stasheff's interest and encouragement. He would like to thank Saunders Mac Lane for a timely letter advocating the use of triples. Finally the author must acknowledge the influence of Victor Gugenheim's paper [3], in which central extensions of Hopf algebras are described by a pair of twisting functions.

\section{REFERENCES}

1. M. Barr and J. Beck, Homology and standard constructions, Sem. on Triples and Categorical Homology Theory (ETH, Zurich, 1966/67) Springer, Berlin, 1969.

2. R. Godement, Topologie algêbrique et thêorie des faisceaux, Actualités Sci. Indust., no. 1252 = Publ. Math. Univ. Strasbourg, no. 13, Hermann, Paris, 1958. MR $21 \# 1583$.

3. V. K. A. M. Gugenheim, On extensions of algebras, co-algebras and Hopf algebras. I, Amer. J. Math. 84 (1962), 349-382. MR 26 \#1340.

4. J. Milnor and J. C. Moore, On the structure of Hopf algebras, Ann. of Math (2) 81 (1965), 211-264. MR 30 \#4259.

5. W. Singer, Extension theory for connected Hopf algebras, Bull. Amer. Math. Soc. 76 (1970), 1095-1099.

Boston College, Chestnut Hill, Massachusetts 02167 